\title{
ECG signal coding methods in digital systems
}

\author{
Tomasz Żentara \\ Military University of Technology \\ Urbanowicza Str. 2, \\ 00-908 Warsaw, Poland, \\ email: tomasz.zentara@wat.edu.pl
}

\author{
Krzysztof Murawski \\ Military University of Technology \\ Urbanowicza Str. 2, \\ 00-908 Warsaw, Poland \\ IEEE Member \# 92707852 \\ email: krzysztof.murawski@wat.edu.pl
}

\begin{abstract}
Article contains an overview of ECG signal coding methods. The presented methods are used to record and present the raw ECG signal in digital systems. The aim of the presentation is to choose the best technique for use in the ECG recording device, currently being developed by the authors.
\end{abstract}

\section{INTRODUCTION}

$\mathrm{T}$ he study of electrical activity of the heart using an electrocardiograph (ECG) has become one of the basic research used in medicine in the diagnosis of many heart diseases over time $[1,2]$. The first ECG recorders used the needle's movements ended with the marker, which marked on the moving tape the electrical indications of the heart. Nowadays, the development of digital systems, as well as the miniaturization of computer systems and microcontrollers allowed to significantly increase the possibilities, while reducing the size and weight of ECGs deices. At the same time, a "revolution" took place, consisting in replacing the paper record for portable memory as well as adding, often even built-in, displays or screen monitors.

The integration of medical and contextual data obtained from the patient into the structure of a single source file or message is very important in case of operating of digital systems used to process medical signals. At present, a number of companies producing ECG monitoring devices have their own methods of coding the signal of cardiac activity [3]. It is worth noticing that standardization organizations have already presented defined file formats and protocols describing medical information [4]. They usually contain elements describing the patient, e.g.: code, name, surname, weight, height, etc., and ECG biosignals or data from the encephalograph (EEG). General characteristics and activities of individual protocols are widely analyzed [57]. One of many examples is the SCP-ECG protocol that integrates ECG, EEG and carbon dioxide (CO2) sensor signals. [6]

This work was not supported by any organization

\section{MOTIVATION}

The article presents and characterizes the standards used in coding biomedical signals - mainly due to the authors' interest in the ECG signal.

The motivation for the work was to present and describe the currently used ECG signal coding standards. What is an important step to develop a prototype of own device used to measure ECG signal, in which selected standards will be implemented and tested.

\section{LITERATURE RESEARCH}

During the preparation of the publication, 16 literature items were analyzed, including 2 books [1,2], 6 articles and publications in scientific journals [3,5-7,11,12], as well as five generally accessible instruction manuals and user instructions $[4,13-16]$.

As a result of literature analysis, the most important standards were specified, including SCP-ECG, MFER, HL7 and DICOM. They have been analyzed in terms of the implementation possibilities in the constructed ECG device. The conclusions from the analysis are included in this publication.

\section{MEDICAL SIGNALS PRESENTATION IN DIGITAL SYSTEMS}

The basic problem identified in the field of bioengineering lies in a different look at the medical device by engineering and medical personnel. Doctors and medical staff are mainly focused on the visualization of the ECG signal. An example of such signal and it specific characteristic fragments as well as time intervals between them is shown in Fig. 1. It is analyzed by doctors who, as a result of its analysis, can determine a number of heart diseases or irregularities of its work.

The article focuses on the engineering area. Therefore, the form and accuracy of the ECG signal recording was more important that the analysis of its shape. The main reason for 


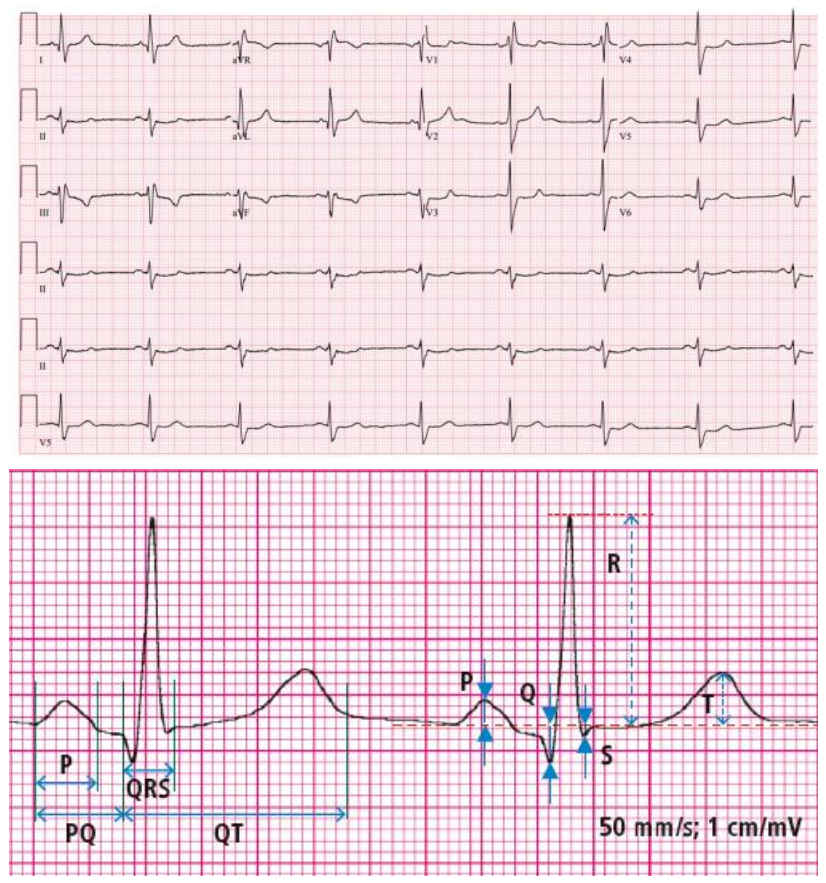

Fig. 1. ECG signal recording along with key elements and their markings $[8,9]$

this interest is the fact, that sensors connected to the patient's body register only an analog electrical signal. The signal presented in this form can not be saved in the memory of the digital device. This problem is solved on the way of conversion to the digital form. The analog / digital converters are responsible for signal conversion. Usually, they are fast 24 bit $\Delta / \delta$ transducers that ensure signal conversion and sample representation accuracy at single bit level. This means in practice that the processing speed is so large that each of the two neighboring samples can differ only at the level of one bit. The signal prepared in this way is stored in the digital device and subjected to standardization and / or normalization.

\section{CONCEPT FOR THE PROTOTYPE DEVICE}

The project of the prototype is based on the use of the Texas Instruments ADS1298 ECG SOC specialized FrondEnd system. The ECG measuring electrodes will be connected to the connector, after which the signal will go through the set of filters and then to the ADS1298 system. This system will convert the analog signal into a digital form so that the evaluation set, equipped with the ARM processor, can read the raw ECG data. Next, for the data presentation the LCD is planned to use, as well as the recording of these data on an removable flash memory. In addition, these data will be sent via a radio link directly to the PC. Hence, so much emphasis on the choice of the appropriate coding standard. The concept device diagram is shown in Fig. 2.

Due to the newly-introduced regulation enforced in Poland related with personal data protection (polish shortcut RODO), for research purposes, it is planned to use mainly
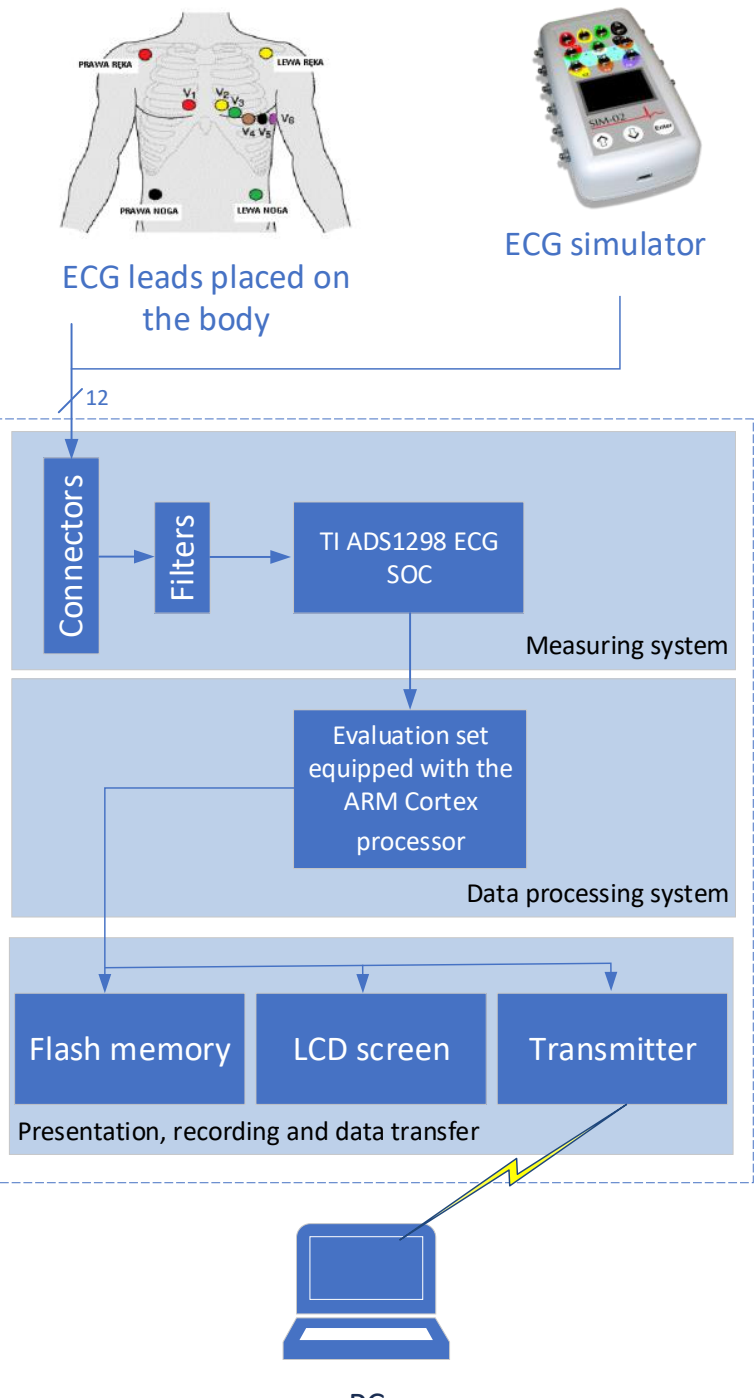

PC

Fig. 2. Concept for the prototype device

artificial patient (ECG simulator), which will be generating the test data, as well as anonymous volunteers.

\section{DESCRIPTION OF THE SPECIFIC STANDARDS}

The first of the described ECG signal coding standards is the Standard Communication Protocol for Electrocardiography (SCP-ECG). It was developed as part of the implementation of the European ECG project in 19891991. In 1993, it was approved as the ENV 1064 standard. Sources report [5] that this standard is now a non-expanding standard, however other sources [6,7] question this statement by saying that it has been revitalized and continues to function only in the new version.

In both cases, the SCP-ECG standard imposes the requirement to use the structural form of cardiological information. This form adapts to the specific pattern of the usually imposed sections. A list of sections for the older SCP-ECG standard is shown in Table 1. The form of the newer standard is shown in Table 2. 
Comparison of Tab. 1 and Tab. 2 shows the difference between the newer (Table 2) and the older (Table 1) version of the SCP-ECG standard. In the newer version, seven optional sections have been added. However, section 4 corresponding to the old standard for the location of QRS complexes (allowing for further subtraction of the reference rhythm to calculate the "residual signal") was reserved in the new version. In the older version of the SCP-ECG standard (Table 1), the section description is presented as follows. As the first one there is a section of indicators, which is a specific list of contents for the entire SCP record and can be used to identify the contents of the record. The next section, containing header information, can consist of up to 35 tags describing a number of patient data, including: identification number, first name, last name, age, weight, height, etc., as well as information on the medicines to be taken, reasons for referral, the apparatus used to measure, along with the software version of these apparatus. In addition, in the header section it is possible to write a text history about the subject of the patient's illness.

The next (in this case optional) section 2 stores information on how to encode the ECG signal, which is recorded in the following sections (5 and 6). In section 2

Tab. 1. The record structure for the older version of the SCP-ECG standard [5]

\begin{tabular}{|c|c|c|}
\hline Name & Field status & Content \\
\hline & Mandatory & $\begin{array}{l}\text { Checksum (CRC) of the entire record } \\
\text { (except this field) - } 2 \text { bytes }\end{array}$ \\
\hline & Mandatory & The entire record size is 4 bytes (unsigned) \\
\hline Section 0 & Mandatory & Pointers to data areas in the record \\
\hline Section 1 & Mandatory & $\begin{array}{l}\text { Header information } \\
\text { - patient data/ECG } \\
\text { - Acquisition data }\end{array}$ \\
\hline Section 2 & Optional & $\begin{array}{l}\text { Huffman tables used in encoding of ECG } \\
\text { data (if used) }\end{array}$ \\
\hline Section 3 & Optional & ECG leads definition \\
\hline Section 4 & Optional & $\begin{array}{l}\text { QRS location (if reference beats are } \\
\text { encoded) }\end{array}$ \\
\hline Section 5 & Optional & $\begin{array}{l}\text { Encoded reference beat data if reference } \\
\text { beats are stored }\end{array}$ \\
\hline Section 6 & Optional & $\begin{array}{l}\text { „Residual signal” after reference beat } \\
\text { subtraction if reference beats are stored, } \\
\text { otherwise encoded rhythm data }\end{array}$ \\
\hline Section 7 & Optional & Global ECG measurements \\
\hline Section 8 & Optional & $\begin{array}{l}\text { Textual diagnosis from the "interpretive" } \\
\text { device }\end{array}$ \\
\hline Section 9 & Optional & $\begin{array}{l}\text { Manufacturer specific diagnostic and } \\
\text { overreading data from the "interpretive" } \\
\text { device }\end{array}$ \\
\hline $\begin{array}{l}\text { Section } \\
10\end{array}$ & Optional & Per lead ECG measurements results \\
\hline $\begin{array}{l}\text { Section } \\
11\end{array}$ & Optional & $\begin{array}{l}\text { Universal statement codes resulting from } \\
\text { the interpretation }\end{array}$ \\
\hline
\end{tabular}

there is also a recoding table for lossless Huffman compression. Another, this time the third section contains a definition of the number of leads used to measure the electrical activity of the heart. The SCP-ECG standard permits the use of up to 255 measuring electrodes that can be distributed over the entire patient's body surface. However, the most common sets are 12 leads for ECG electrodes. For the most commonly used electrode sets, preliminary code lists of lead systems have been prepared as standard. It is also worth mentioning that section 3 contains information about the length and simultaneity of records for individual leads, so that the raw signal recording from individual electrodes can have different lengths and does not have to be made at the same time. Section 4 is responsible for storing position indicators for representative QRS wave units recorded in section 5. Section 6 in the SCP-ECG standard is intended for storing the signal after subtracting the representative units recorded in section 5 or the raw heart rhythm electric signal read from the cardiograph electrodes. The filling of section 7 requires more advanced devices that allow detection of the remaining electrocardiogram waves. For storing data for section 8 , it is required to use devices equipped with diagnostic algorithms to classify the evolution of the heart. Sections 7 to 11 are classified for implementation in advanced electrocardiographs equipped with algorithms for recording interpretation, and also in efficient computer components.

Compared with the older version of the SCP-ECG standard (Table 1), the main changes in the newer version (Table 2) are the disappearance of the bimodal compression scheme and the subtraction of representative units that apply to sections 4 to 6 . Lossless compression (Huffman coding) of short-term ECG data rhythms (section 6) and representative 'type 0 ' units (section 5) is allowed. Additionally, to simplify decoding, it is recommended in the new standard to save all ECG signal data as uncompressed, in the form of a series of integer lengths with a sign. However, in exceptional situations, e.g.: slow network data transmission or limited wireless connectivity, for mobile devices the standard continues to use Huffman coding and the differentiation of representative signals.

As mentioned earlier, in the SCP-ECG version 3.0 standard, new sections have been added to the record, and so the standard now provides the option of storing long-term ECG signal (section 12), e.g. for 3-lead ECG at 200 samples / $\mathrm{s}$ with a 16-bit resolution, continuous recording up to 40 days is possible. The format of section 12 is very similar to the ISHNE [11] format used to record data in Holter devices. Additional sections 13 and 14 have been introduced to support stress test records and drug trials, where section 13 contains metadata, or representative assemblies (or pointers for selected reference units), and section 14 contains several selected medium or short-term ECG sequences. In section 15 it is possible to store several predefined global measurements and lead measurements together with annotations to them. 
Tab. 2. The record structure for the SCP-ECG standard in the newer version 3 [10]

\begin{tabular}{|c|c|}
\hline $\begin{array}{l}\text { Field } \\
\text { status }\end{array}$ & SCP-ECG record structure \\
\hline Mandatory & $\begin{array}{l}\text { Checksum (CRC) of the entire record (except this field) } \\
\qquad-2 \text { bytes }\end{array}$ \\
\hline Mandatory & The entire record size is 4 bytes (unsigned) \\
\hline \multirow[t]{2}{*}{ Mandatory } & (Section 0$)$ \\
\hline & Pointers to data areas in the record \\
\hline \multirow[t]{2}{*}{ Mandatory } & (Section 1) \\
\hline & $\begin{array}{l}\text { Header information - patient data/ECG } \\
\text { Acquisition data }\end{array}$ \\
\hline \multirow[t]{2}{*}{ Optional } & (Section 2) \\
\hline & Huffman tables used in encoding of ECG data (if used) \\
\hline \multirow[t]{2}{*}{ Mandatory } & (Section 3) \\
\hline & ECG leads definition \\
\hline \multirow[t]{2}{*}{ Mandatory } & (Section 4) \\
\hline & Reserved for legacy SCP-ECG versions \\
\hline \multirow[t]{2}{*}{ Optional } & (Section 5) \\
\hline & $\begin{array}{c}\text { Encoded type } 0 \text { reference beat data (if reference beat is } \\
\text { stored) }\end{array}$ \\
\hline \multirow[t]{2}{*}{ Optional } & (Section 6) \\
\hline & Short-term ECG rhythm data \\
\hline \multirow[t]{2}{*}{ Optional } & (Section 7) \\
\hline & Global ECG measurements \\
\hline \multirow[t]{2}{*}{ Optional } & (Section 8) \\
\hline & Textual diagnosis from the "interpretive" device \\
\hline \multirow[t]{2}{*}{ Optional } & (Section 9) \\
\hline & $\begin{array}{l}\text { Manufacturer specific diagnostic and overreading data } \\
\text { from the "interpretive" device }\end{array}$ \\
\hline \multirow[t]{2}{*}{ Optional } & (Section 10) \\
\hline & Per lead ECG measurements \\
\hline \multirow[t]{2}{*}{ Optional } & (Section 11) \\
\hline & $\begin{array}{c}\text { Universal statement codes resulting from the } \\
\text { interpretation }\end{array}$ \\
\hline \multirow[t]{2}{*}{ Optional } & (Section 12) \\
\hline & Long-term ECG rhythm data \\
\hline \multirow[t]{2}{*}{ Optional } & (Section 13) \\
\hline & $\begin{array}{l}\text { Stress tests, drug trials and protocol-based ECG } \\
\text { recordings metadata }\end{array}$ \\
\hline \multirow{2}{*}{$\begin{array}{l}\text { Depends } \\
\text { on section } \\
13\end{array}$} & (Section 14) \\
\hline & Selected ECG sequences repository \\
\hline \multirow[t]{2}{*}{ Optional } & (Section 15) \\
\hline & Beat-by-beat ECG measurements and annotations \\
\hline \multirow[t]{2}{*}{ Optional } & (Section 16) \\
\hline & Selected ECG beats measurements and annotations \\
\hline \multirow[t]{2}{*}{ Optional } & (Section 17) \\
\hline & Spikes measurements and annotations \\
\hline \multirow[t]{2}{*}{ Optional } & (Section 18) \\
\hline & Additional ECG annotations \\
\hline
\end{tabular}

The recorded sequences may refer to single beat selected by a doctor or a sequence typing algorithm (depending on the progress of the device / selected scenario), or to the whole set of beats from one / many time windows recorded in longterm section 12, short-term section 6 or data from section 14 . The next element is section 16 , which provides solutions for storing another set of measurements and annotations than those recorded in the previous section. The structure and format of section 16 are the same as in section 15 , so you can say that both sections are complementary with each other. However, in section 16 there is no restriction on determining the time window. Section 16 is preferred for storing heart beat measurements if "beat by beat" measurements are not required (if section 15 is not present). Another optional section, with number 17, has been designed to provide support for predefining and storing large global and / or prelead spike measurements and annotations to them.

In addition, it can save spike by spike measurements into one or more measurement tables. The main purpose of Section 18 ("Additional ECG Annotations") is to provide a solution for storage of any type of manually or automatically created annotations that have not been systematically stored in Sections 7, 8, 10, 11 and 15 to 17. These include: start (and end) of the original rhythm or atrial fibrillation, identification of the pacemaker spike which was not included in Part 17, and measurements not provided in section 15 and 16 (or several measurements, such as QT interval, neither section 15 nor section 16), manual annotations of more complex cases with different types of abnormal QRS complexes (LBBB aberration etc.) and $\mathrm{P}$ waves (AV dissociation, etc.), as well as annotations for example about the noise level in a given lead, etc.

The second standard, the Medical Waveform Format Encoding Rules (MFER), in contrast to the previously described SCP-ECG standard, is used not only to encode ECG signals, but also any other medical signals. Although the MFER standard is not dedicated to use only in electrocardiography, it is in this area of interest that the most reports on its use are made [5, 12]. It is said that standard was proposed in 2004 by the Japanese standardizing organization in place of numerous signal coding methods used by manufacturers of measuring apparatus. The main task for this standard was to unify the record of raw medical data and as much as possible simplification of generalpurpose medical information exchange systems. Figure 3 shows the information model used in the MFER standard. As you can see, it assumes maximum simplicity of implementation, all in order to achieve transparency of the record and wide applicability (from simple applications / devices to advanced and nested hospital information systems). As you can see, Figure 3 shows the general view on the composition of the full message packet sent in the MFER standard. Analyzing more closely Fig. 3, it can be noticed that the MFER message consists of four main groups, i.e.: descriptive information about the observations, appropriately recorded ECG waveform, information about the patient, as well as an additional description. Data on the ECG signal read are further extended to three parts, i.e.: channel information, frame information and raw data read from the electrocardiograph electrodes. In the further part of the article, a more detailed description of the frame layout regarding to the data read from the electrocardiogram. It is worth noting that the MFER standard is intended to encode only signals, while the coding of other medical data (e.g. 


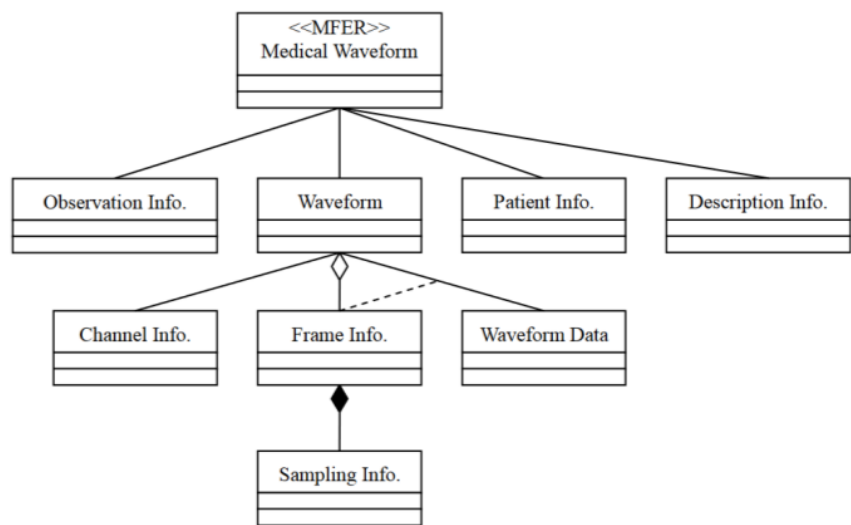

Fig. 3. The signal information model used in the MFER standard [13]

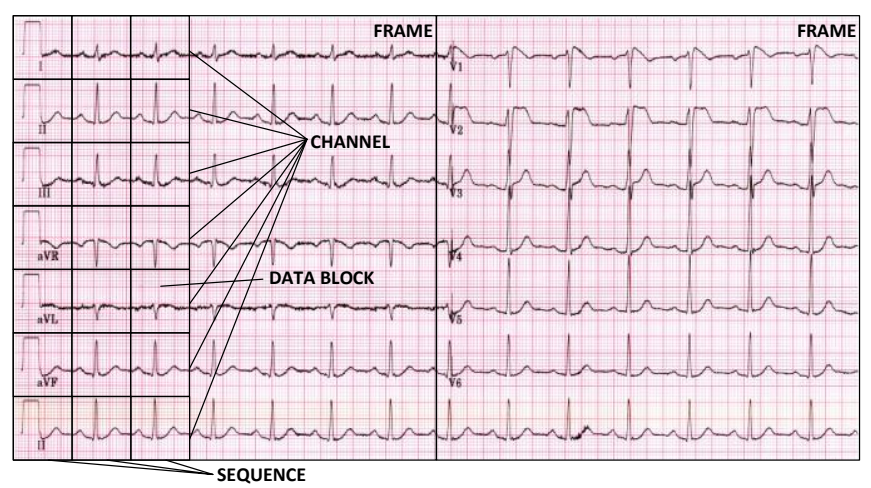

Fig. 4. Frame attributes in MFER [13]

images) that often accompany these signals MFER leaves other standards.

The MFER standard is used for coding multidimensional signals, which are divided into time frames (Figure 4). In terms of the frame, we can distinguish main attributes, which are: frame description and description of sampling. The sampling description consists of data about the frequency used and the sample resolution. The description of the frame contains information about the synchronization of signals, as well as descriptions of the three main components of the frame, i.e.: data blocks, recording channels and sequences (Figure 4).

The header and the measured signal must be coded according to the MFER rules. They should consist of type specifications (attributes such as number, primary tag and class), length specification - defining the length of the data section and value string, which is the basic information content stored in the file. The header and signal encoded in the form of MFER are shown in Figure 5.

Fig. 5 presents the general signal coding method used in the MFER standard. At this point, the question should arise how it affects the channels, sequences and data blocks, i.e. how to place data in the frame to meet the coding rules in the MFER.

To illustrate the above consideration, it is worth to transfer attention to Figure 6, which presents the detailed arrangement of the data values in the frame. As you can see in Figure 6, three channels are available (i.e. three ECG electrode leads). The recorded signal has been divided into

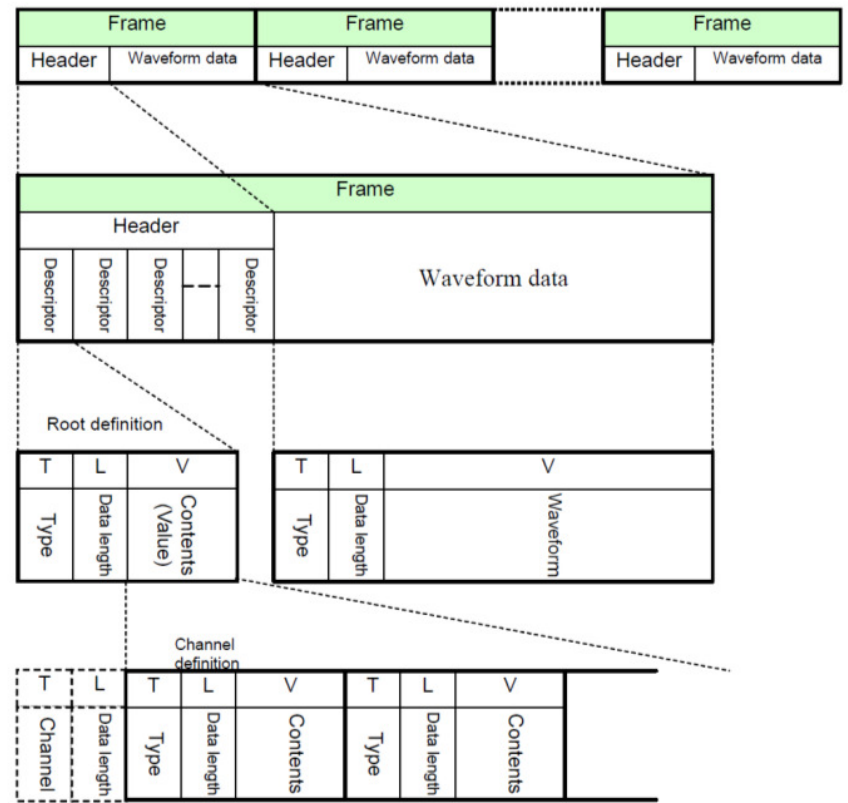

Fig. 5. Header and signal presented in the MFER encoding format [14]

four sequences, so in one frame we have to save twelve blocks of data. In addition, each block consists of five sampled signals / "beats". The result of the data arrangement in the frame will be a string consisting of a header with saved (as in Fig. 6) information about the frame and raw data from the electrodes.

For example, let suppose that sampling is done with a period of $4 \mathrm{~ms}$, so the sampling frequency is $250 \mathrm{~Hz}$, which gives us $20 \mathrm{~ms}$ for one block of recorded data ( $4 \mathrm{~ms} * 5$ samples $=20 \mathrm{~ms})$. Since there are four sequences in one frame, the waveform data for each channel is $80 \mathrm{~ms} /$ frame $(20 \mathrm{~ms} * 4)$.

All complexities related to the MFER standard are widely described in the documentation available on the manufacturer's website, although not all documents are in English (a large part of the documentation is available only in Japanese).

By assumption, MFER is to meet the principle of maximum flexibility as well as the simplicity of implementation. The tags contained in the MFER have

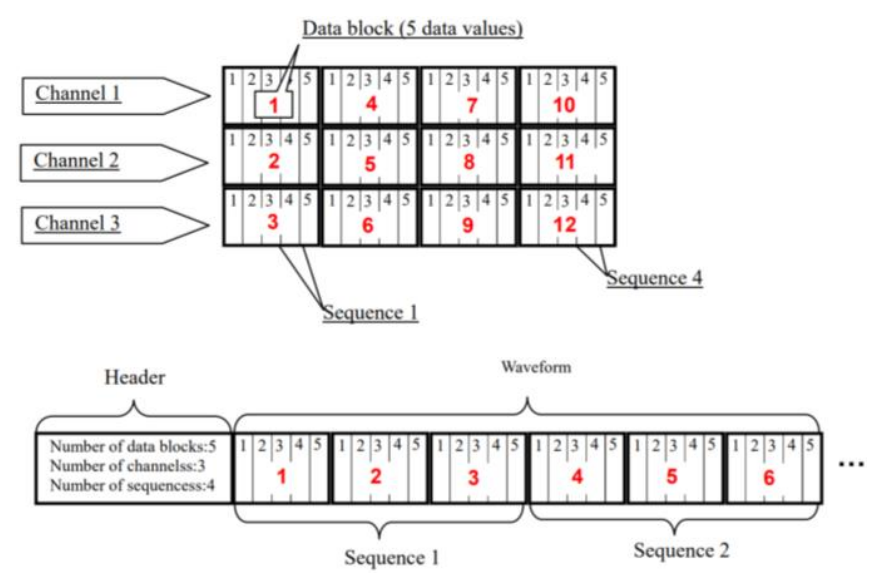

Fig. 6. Data layout in the MFER standard frame [14] 
predefined default values that can be changed / adjusted when using a custom one. The MFER standard can be used to describe the signals obtained from a 12-lead electrocardiograph, a 24-hour continuous Holter recording, a vectocardiogram, stress tests, electroencephalogram, etc. Another standard that can be used to encode ECG signals is Health Level Seven (HL7), and more specifically ANSI / HL7 V3 Annotated ECG (HL7 aECG). In general, HL7 can be described as the standard for electronic information exchange provided for medical environments. The standard was developed by HL7, which was established in 1987. In addition, part of the standard name, which is "level seven" can be referred to the ISO / OSI model, and more specifically the seventh layer of the model - the application layer (it can be said that HL7 uses the "transparency" of the following layers).

The Annotated ECG HL7 standard was developed in response to the initiative of the American Food and Drug Administration (FDA) regarding digital recording of ECG signals. A specific background to deriving this standard was the need to review and evaluate the collected ECGs in clinical trials of drugs. Until now, ECG entries with annotations were delivered to the FDA mainly in paper form, so the next logical step was to take the initiative of digitally sending ECGs with annotations in a standardized format / standard. That's why FDA, sponsors, key laboratories and manufacturers of medical devices have worked together and with HL7 to create a standard that meets all their needs.

The HL7 V3 standard is based on an object-oriented approach and the message structure is designed using the XML markup language. Each saved ECG file must be marked with a unique name (Unique ID), and should contain annotations about the stored ECG signal, which are later verified by the FDA. Due to the fact that annotations are one of the key elements of ECG recordings, this standard is known under the name Annotated ECG in XML.

Message saved in the HL7 aECG standard must be properly encoded, i.e. similar to SCP-ECG where sections are provided, here are the required and optional tags. Figure 7 shows an example of XML schema that contains a minimal description of an aECG message. A detailed description of how to implement individual tags can be found in the extensive documentation available on the manufacturer's website. Analyzing Fig. 7, which describes the absolute minimum to be met, it is clear that there is no information about the value of the recorded ECG signal. Therefore, Figure 8 shows a fragment of the XML schema, which should be added to the diagram shown in Figure 7. This fragment is responsible for storing data taken from the ECG electrode lead (in this case, lead number 1). In the first phase of the project, the recorded ECG signal was stored directly in the structure of the XML schema, and more specifically in the section described by the tag $<$ digits $></$ digits $>$. However, there were cases where the message saved in this way took up 9 GB disk space. To overcome the problem of excessive message size, the aECG standard was slightly modified and the part strictly responsible for storing data in the XML schema was separated into a separate file. In this

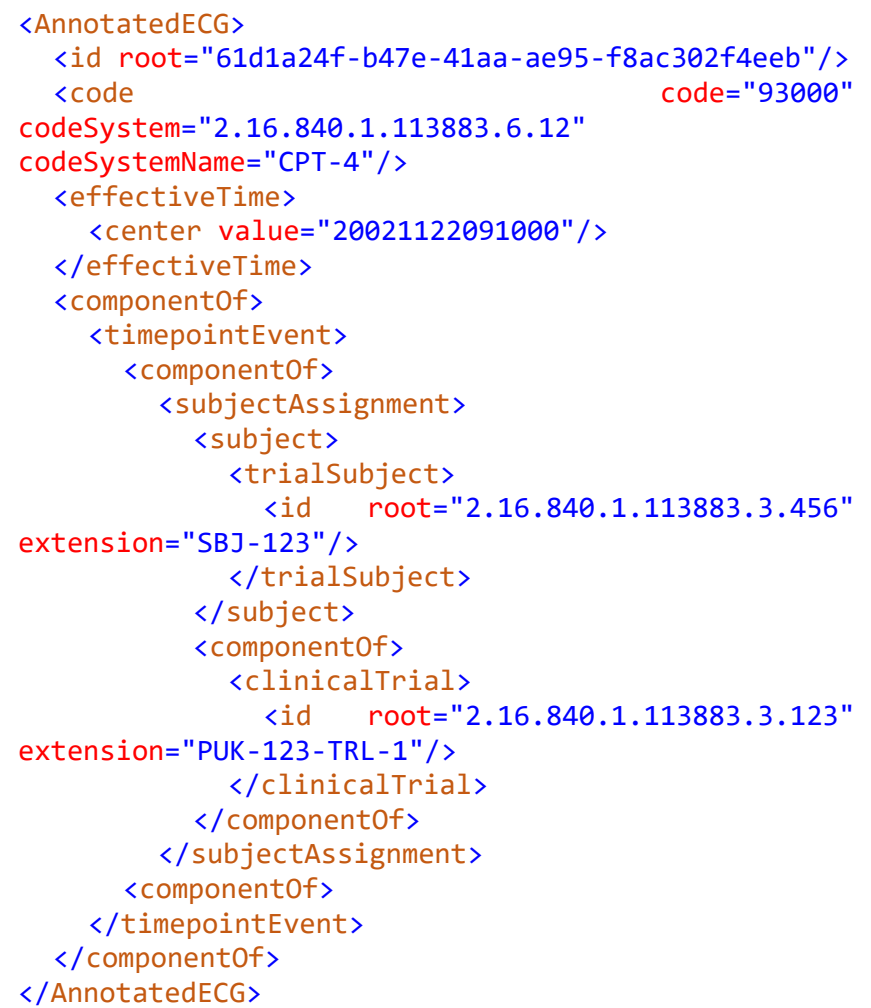

Fig. 7. The minimum set of information that an aECG message must contain [15]

way, the raw ECG signal was transferred to a binary file, whereas in the XML schema there is only an index/pointer on the data file (example - selection in Fig. 8). Figures 7 - 9 shows the record of the ECG signal and the minimal XML schema for HL7 aECG, however, it was mentioned earlier that this is the standard of "annotations", which are further analyzed by the FDA and that is where the greatest emphasis is placed on them. The implementation documentation available on the manufacturer's website contains a number of examples of how to enter annotations, what field codes must

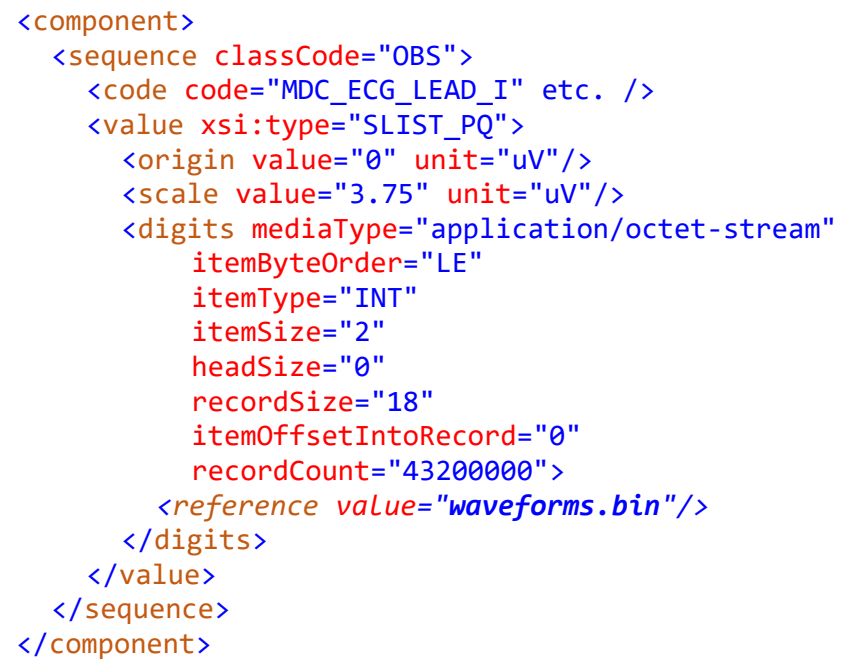

Fig. 8. Pointer on a binary file with a raw ECG signal [15] 


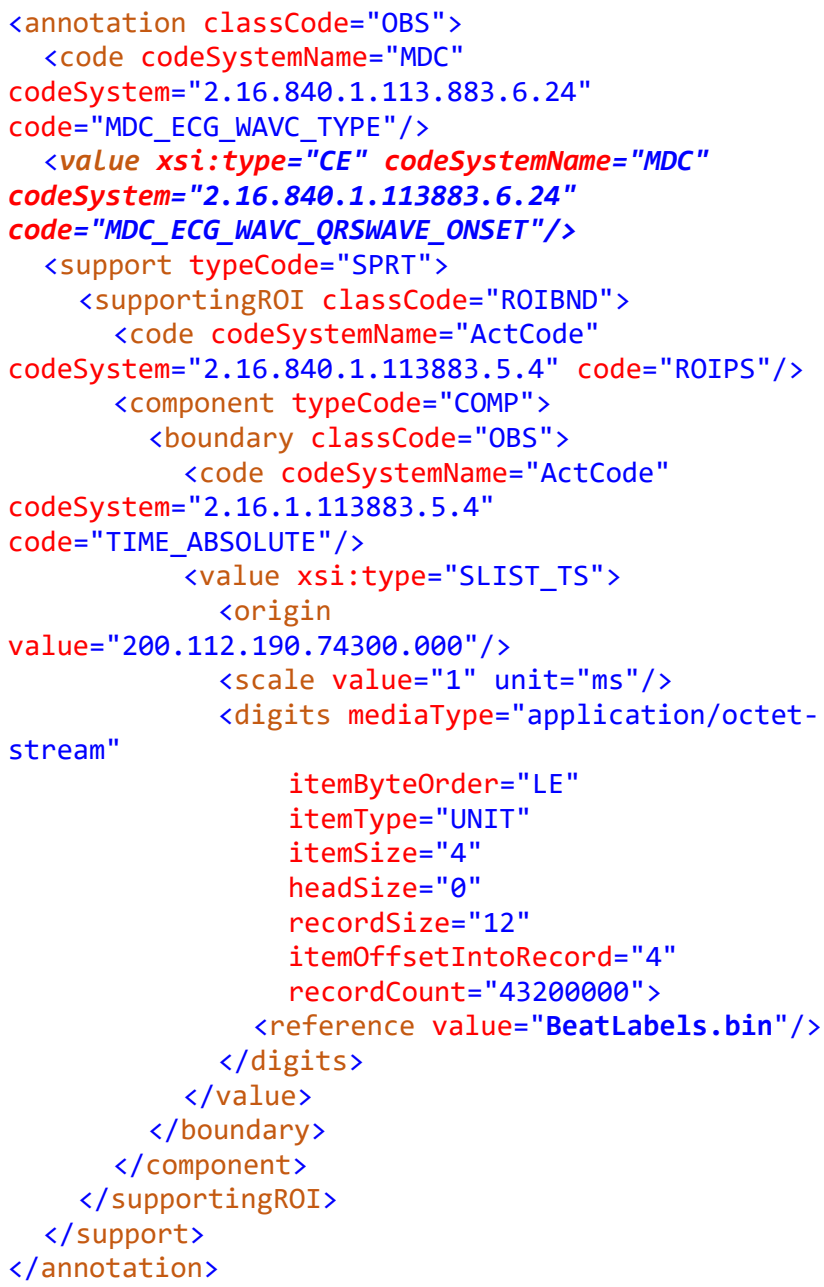

Fig. 9. Annotation about the QRS wave set for the HL7 aECG scheme [15]

be included in the relevant tags, and ready-made XML schemas of popular annotations. An example here is Figure 9 describing the QRS wave set. This is an example of a ready annotation that can be attached to the aECG XML schema. In addition, the selection in Figure 9 indicated a field with a code saying that we are dealing with a QRS wave and the pointer on the binary file in which the selected ECG signal was saved.

The next, listed immediately after HL7, is the standard published in 1993, and in 1995 approved in Europe Digital Imaging and Communications in Medicine (DICOM). According to the mentioned name, this standard is intended for management, archiving, printing and data transmission in the field of medical imaging. Adapted on a large scale in information systems that process medical images such as $\mathrm{x}-$ ray pictures, magnetic resonance imaging (MR), computed tomography (CT), etc. [12]. However, according to Supplement No. 30 of the DICOM standard documentation, it can send non-pictorial data, which are related to the patient (i.e. ECG signal or other signal describing life parameters based on the recording of curves). Additionally, thanks to the introduction of time stamps, correlations between digital

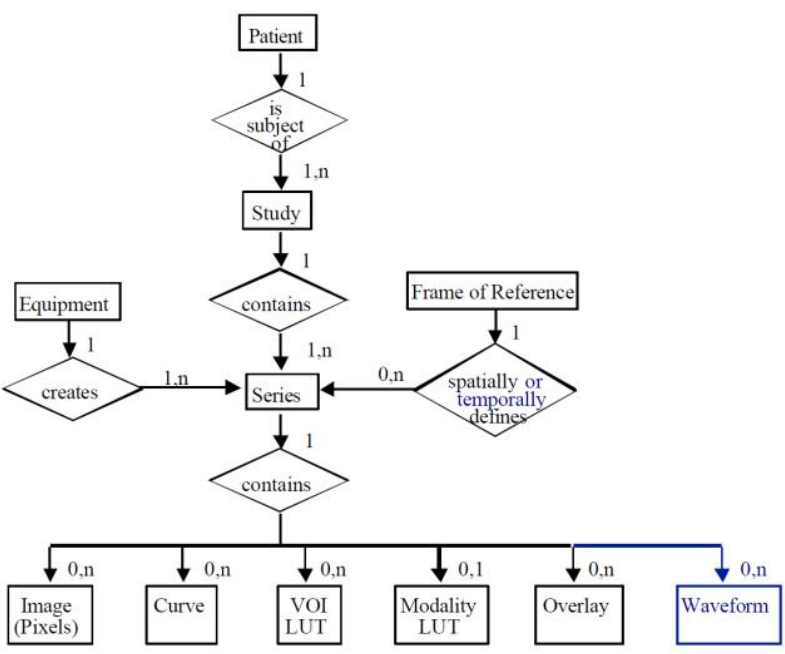

Fig. 10. Information model for data storage in the DICOM standard [16]

images can be visualized, e.g. in the angiographic examination, the heart pressure can be recorded simultaneously. Fig. 10 presents the information model for the DICOM standard, which in the final part may contain a number of different object models, including the blue model called "waveform" which contain curves (ECG or other). The specified model can be called a unit of information about the curve. Each such unit contains a number of technical parameters associated with the attributes of the recorded wave, as well as recorded samples of the raw signal. The exact structure of the curve information model is shown in Figure 11. Each such object contains information about the time in which the signal was acquired and a number of multiplex groups, each group is determined by digitizing with the same clock. The timing frequency for the clock can be defined for each multiplex group. By going down below, a group can contain one or more recording channels with a full technical definition,

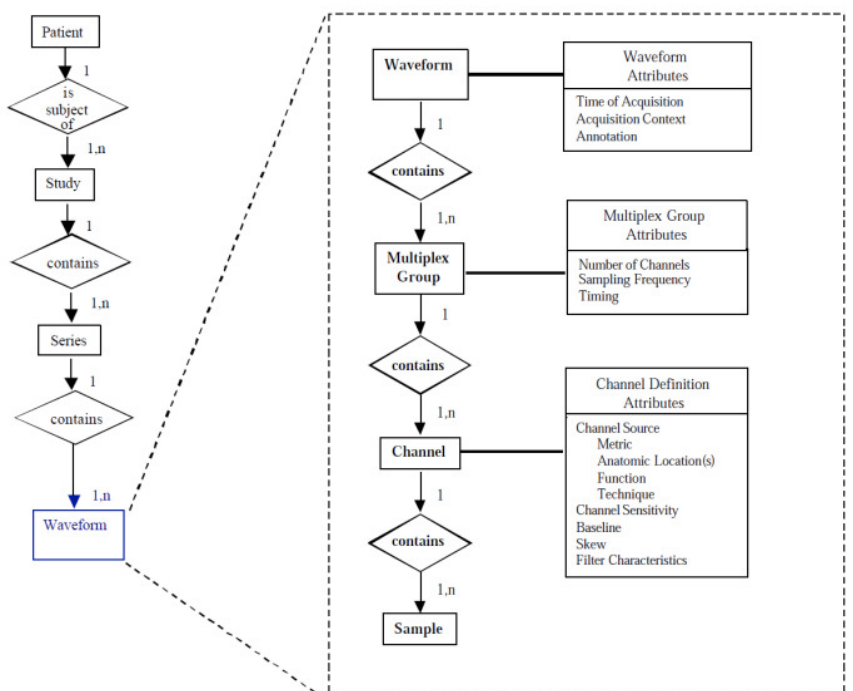

Fig. 11. Waveform information model for the DICOM standard [16] 
moreover, each channel has its own separate set of digital wave samples. Additionally, when analyzing Fig. 11 it can be seen that in the "wave curve" object we can define more than one multiplex group, which gives us the opportunity to register two or more types of multi-channel signals (such as ECG and heart pressure). It is also worth mentioning that in supplement 30 of the documentation of the DICOM standard, it was specified that the definition of the "waveform" object is harmonized with the semantic structure of the HL7 standard, including the definition attributes for synchronously acquired channels. Thanks to the solution, which is based on a common object model, it is easier to share and transcode between the HL7 and DICOM standards.

\section{Telemedicine Conclusions}

Analyzing the specified ECG signal coding standards a specially for future use in the constructed device, it is worth focusing on the SCP-ECG standard, which is designed and used exclusively to handle signals from ECG devices. In addition, the built-in ability to compress a lossless transmitted signal can be a decisive element when using it in the field of telemedicine, or long-term measurements, where the data transmission speed or capacity of storage media device is limited. However, it is also worth considering the HL7 aECG standard, which ensures wider interoperability, and additionally the extracted file with saved raw data is an inspirational element for the application of lossless data compression algorithms.

\section{REFERENCES}

[1] Szczeklik A., Gajewski P., Interna szczeklika 2015, Medycyna Praktyczna, Kraków, 2015

[2] Houghton A. R., Gray D., EKG jano i zrozumiale, $\alpha$-medica press, 2014

[3] Kligfield P. and others, Recommendations for the Standardization and Interpretation of the Electrocardiogram, Journal of the American College of Cardiology Volume 49, Issue 10, 13 March 2007, Pages 1109-1127

[4] Norma PN-EN 1064

[5] Tadeusiewicz R. „Informatyka medyczna”, Instytut Informatyki UMCS, Lublin, 2011

[6] Mandellos G. J., Koukias M. N., Styliadis I. S., Lymberopoulos D. K. e-SCP-ECG+ protocol: An expansion on SCP-ECG protocol for health telemonitoring-Pilot implementation. Int. J. Telemed. Appl. 2010;2010:1-17

[7] Rubel P., Pani D., Schloegl A., Fayn J., Badilini F, Macfarlane P. W., Varri A. SCP-ECG V3.0: An Enhanced Standard Communication Protocol for Computer-assisted Electrocardiography, Computing in Cardiology 2016; 43:309-312.

[8] https://adst.mp.pl/img/articles/kardiologia.mp.pl/ekg/kurs/ekg-2-1.jpg

[9] http://www.imreference.com//rsrc/1467898020602/cardiology/ekg/ Screen\%20Shot\%202016-01-18\%20at\%202.04.32\%20PM.png

[10] http://webimatics.univ-lyon1.fr/scp-ecg/index.php? controler $=$ Home\&action $=$ services

[11] Badilini F., The ISHNE Holter Standard Output File Format, Francja

[12] Rune Fensli, Evaluation of international standards for ECG-recording and storage for use in tele-medical services, 2006

[13] Medical Waveform Format Encoding Rules - Part 1 http://www.mfer. org/doc/MFER Part1 Ver105.pdf

[14] Medical Waveform Format Encoding Rules - Part 3-1 http://www. mfer.org/doc/JPACS-2013-S001.pdf

[15] Brown B. D., Baldini F., HL7 aECG Implementation Guide Final March 21, 2005

[16] Digital Imaging and Communications in Medicine (DICOM) Supplement 30: Waveform Interchange 